\title{
Social intervention for British Pakistani women with depression: randomised controlled trial ${ }^{\dagger}$
}

Richard Gater, Waquas Waheed, Nusrat Husain, Barbara Tomenson, Saadia Aseem and Francis Creed

\section{Background}

British Pakistani women have a high prevalence of depression. There are no reported psychosocial interventions for depression in ethnic minorities in the UK.

\section{Aims}

To determine the efficacy of a social group intervention compared with antidepressants, and whether the combination of the two is more efficacious than either alone.

\section{Method}

A total of 123 women with depression participated in the primary care-based cluster randomised controlled trial (ISRCTN19172148). Outcome measures were severity of depression (Hamilton Rating Scale for Depression), social functioning and satisfaction at 3 and 9 months.

\section{Results}

Greater improvement in depression in the social intervention group and the combined treatment group compared with those receiving antidepressants alone fell short of significance. There was significantly greater improvement in social functioning in the social intervention and combined treatment groups than in the antidepressant group at both 3 and 9 months.

\section{Conclusions}

Pakistani women with depression found the social groups acceptable and their social function and satisfaction improved if they received social treatment compared with the receipt of antidepressants alone.

\section{Declaration of interest}

W.W. has received a research grant from Servier and support for educational, travel support and/or speaker fees from AstraZeneca, Eli Lilly, Sanofi-Aventis, Bristol-Myers Squibb, Janssen Cilag and Wyeth. N.H. has received support for educational programmes, travel support and/or speaker fees from Astrazeneca, Eli Lilly, Lundbeck, Sanofi-Aventis, BristolMyers Squibb, Janssen Cilag and Wyeth. F.C. has received speaker fees from Eli Lilly.
South Asian women living in the UK, particularly of Pakistani family origin, have a higher prevalence of depression, suicide and self-harm than White women. ${ }^{1-7}$ Only $10 \%$ of south Asians with depression are prescribed medication or are referred to psychiatric services, ${ }^{8}$ and those who do receive antidepressants are prescribed lower doses for shorter durations than White people., ${ }^{9,10}$ Other than psychoeducational interventions on patients' perspectives and help seeking ${ }^{11-13}$ there are no reported interventions for ethnic minorities with depression in the UK. Our research indicates that British Pakistani women with depression lack social support and experience marked difficulties particularly in marital and close relationships. ${ }^{1,2}$ They lack fluency in English and the resources to obtain help. This formed the theoretical basis for developing a social group intervention using the Medical Research Council (MRC) framework for complex interventions. ${ }^{14}$ The first step in the development tackled the problems of recruiting socially isolated British Pakistani women with depression to the groups and their response to attending a series of social groups. ${ }^{15}$ In the present trial we aimed to test the feasibility, acceptability and efficacy of social groups for British Pakistani women with depression. First, we hypothesised that social intervention is superior to protocol-guided antidepressant treatment, and second that social intervention combined with protocol-guided antidepressant treatment has greater benefits than either social intervention or antidepressant treatment alone. These results would later help design a larger trial including cost-effectiveness of a social group intervention.

†See editorial, pp. 172-173, this issue.

\section{Method}

\section{Participants}

Six general practices, with high proportions of British Pakistanis in the north-west of England were recruited. Presentations were made to voluntary organisations for ethnic minorities, key persons in the Pakistani community, and staff of the practices to gain their support. Multilingual invitation posters were displayed to encourage recruitment. The study timetable was planned to avoid recruitment, interventions and assessments during Muslim festivals and school holidays.

We consecutively screened British Pakistani women aged between 16 and 65 years attending the practices, communicating with them in English, Urdu or Punjabi. Those with intellectual disabilities or severe mental illness or planning a visit abroad during the next 6 months were excluded. Patients already receiving antidepressants or any psychological intervention were not excluded.

In a two-phase procedure, the Self-Reporting Questionnaire $(\mathrm{SRQ})^{16,17}$ was administered to identify probable cases. Those scoring 7 or more were interviewed using the Clinical Interview Schedule - Revised (CIS-R), a structured clinical interview, ${ }^{18}$ to confirm a diagnosis of depression.

Independent cluster randomisation using www.randomization. com was carried out at the level of general practice (six clusters) to avoid contamination between the intervention arms. Participants with depression were allocated either to: (a) social intervention; (b) protocol-guided antidepressant treatment; or (c) a combination of both interventions (trial registration: ISRCTN19172148).

Before starting the interventions, group facilitators visited the homes of participants allocated to the social intervention to 
inform them about group allocation. Participants in the antidepressant treatment arm received an appointment letter from their general practitioner (GP) to discuss antidepressant prescription. Participants in the combined treatment group received both home visit and invitation letter. Three- and ninemonth follow-up assessments were arranged at a venue of the participant's preference.

Written informed consent was sought at screening and then again before randomisation. Ethical approval was received from Tameside and Glossop, and East Lancashire Research Ethics Committees (04/Q1402/2).

\section{Interventions}

A multidisciplinary team of psychiatrists, psychologists, British Pakistani mental health workers and service users at the University of Manchester developed the social intervention based on MRC framework for complex interventions. ${ }^{14}$

\section{Social intervention}

The culturally sensitive social intervention was designed to address social difficulties, isolation and poor access to primary care. It was delivered by two facilitators as a stand-alone intervention, and in collaboration with the GP when combined with antidepressant use.

During phase I of intervention development it was suggested that appropriate intervention should begin with the development of informal networks that engage these women in social contacts, and link them with appropriate treatment. The social intervention involved 10 women attending facilitated groups at a local community centre for weekly sessions over 10 weeks. Group activities were developed in consultation with the voluntary organisations for ethnic minorities and were informed by our pilot study. ${ }^{15}$ At the first session, the participants choose from a list of indoor and outside activities for subsequent sessions. A psychoeducation session provided information about depression including its nature, symptoms, causes and treatment.

To ensure cultural sensitivity, participants were collected by taxi accompanied by a female transport facilitator. The groups took place in a culturally acceptable venue with provision of childcare facilities. Participants were addressed and greeted in a traditional manner and at the beginning of each session they were reminded about confidentiality as this had previously been cited as a major reason for non-engagement with services. ${ }^{15}$ The selection of group activities was culturally appropriate and food was provided at the end of each session.

\section{Antidepressant intervention}

Participants randomised to the antidepressant intervention were specifically invited and offered treatment by their GP, who was trained in the treatment protocol and monitoring following National Institute for Health and Clinical Excellence (NICE) guidelines. $^{19}$

\section{Combined intervention}

In the combined intervention, participants were invited to attend social groups as well as being offered being antidepressant treatment.

\section{Training and supervision of group facilitators}

Four multilingual graduate women were trained as group facilitators. Based on our pilot study, ${ }^{15}$ a manualised, 5-day training programme was developed and delivered by psychiatrists experienced in cross-cultural mental health, a group facilitation skills trainer and a British Pakistani mental health worker. Training included lectures, case scenarios and role play covering principles of randomised trials, communication and group facilitation skills. The format and content of the group sessions were described with particular emphasis on developing empathy, maintaining confidentiality and facilitating engagement. Each facilitator demonstrated their competence by facilitating a mock group.

During the trial, facilitators received weekly supervision from a psychiatrist (W.W.). Midway through the social intervention the facilitation skills trainer conducted a feedback and advisory session with the facilitators to address any problems and issues in running the groups. They were also encouraged to interact among themselves, seek advice and share their experiences. Four local British Pakistani women were trained as transport facilitators. They accompanied participants from their home to the centre and back, communicated with participants and encouraged attendance.

The participating GPs allocated to the antidepressant or combined treatment arm received 1-hour training based on NICE guidelines. They were provided with plastic-coated depression treatment algorithms to refer to and electronic reminders for computerised case records or prompt cards inserted into the paper-based case notes.

\section{Measures}

Assessments were carried out at baseline, 3 months (completion of the intervention) and 6 months post-intervention ( 9 months after baseline). Baseline assessment included demographic data, psychiatric history, Hamilton Rating Scale for Depression (HRSD) score ${ }^{20}$ and a measure of social functioning specifically created for British Pakistani women. ${ }^{21}$ The HRSD rates depressive symptoms on a scale from 0 to 48 ; higher scores indicate greater severity of depression. The self-rating social functioning questionnaire rates the degree of difficulty in completing ten daily functions from 0 (no difficulty) to 4 (often can't do the task), with a total score ranging from 0 to 40 .

The baseline measures were repeated along with self-rated satisfaction with treatment at 3 and 9 months, based on a threepoint ordinal scale derived from Verona Service Satisfaction Scale ${ }^{22}$ that rated six items from 1 (definitely not satisfied) to 4 (very satisfied) (maximum possible range: 6-24).

Previously translated and validated measures were used, and those being used for the first time in Urdu were translated following a standard protocol. ${ }^{23}$ Owing to expected limited literacy, all questionnaires were read to the patients in a standardised way. Three British Pakistani postgraduate researchers received initial 2-week training on the use of assessment schedules and culturally sensitive research methods focusing on recruitment, consent and trial procedures. Researchers independent of those providing the intervention and masked to the intervention allocation and adherence undertook outcome assessments.

\section{Qualitative data collection}

Qualitative data were collected initially through a feedback form completed jointly by the group facilitators and individual participants at the end of each session, specifically documenting participants' quotes. After the 9-month follow-up assessments, a focus group of eight participants representing all three treatment arms was organised and data were collected until saturation was reached within the focus group session. 


\section{Power calculation}

The change in HRSD score was used as the primary outcome variable. Sample size estimation indicated that 40 women in each of the three intervention arms would have $89 \%$ power if the difference in mean HRSD scores were 4 . The power will be reduced to $65 \%$ if the difference is 3 , assuming a fixed within-group standard deviation of 5.0 for all comparisons, and using a two-sided significance level of 0.025 (using the Bonferroni correction, dividing the nominal alpha by two, to allow for two between-group comparisons).

\section{Statistical methods}

We followed the CONSORT guidelines for cluster randomised controlled trials. ${ }^{24}$ The main analyses were based on intentionto-treat principles. Supplementary analyses included allowance for loss to follow-up. ${ }^{25}$ For categorical variables, the numbers and percentages of patients in each group are presented and were compared using the $\chi^{2}$-test. Means and standard deviations are presented for continuous variables at baseline, and were compared using one-way ANOVA followed by Bonferroni corrected pairwise comparisons between groups. For changes from baseline to follow-up on continuous variables, intervention effects were compared using linear regression with the covariates of baseline score and treatment group (as dummy variables for each active social intervention $v$. antidepressants only) using the Stata command regress (Stata version 9 for Windows). A second regression was used to compare combined treatment with the social intervention. Robust standard errors are calculated specifying GPs as the six clusters. Means and standard deviations in each group, and 95\% confidence intervals and significance of the intervention effects are presented. We also present an analysis of the HRSD where the data were analysed by patient rather than practice.

\section{Results}

\section{Participant flow}

Between September 2004 to April 2005 in Manchester and from May 2005 to October 2005 in Accrington, 488 patients from six GP clusters (range 63-118 per cluster) completed the SRQ (Fig. 1). The 252 potential cases that scored 7 or more on the SRQ were invited for CIS-R interview until the number of participants with depression needed per cluster was achieved. Of these, 83 refused participation or were excluded for other reasons and 169 were interviewed, of whom 148 had depression. A further 25 subsequently refused, leaving 123 participants who were cluster randomised into three treatment arms.

There was no significant difference between the 25 individuals refusing participation and those who consented, with respect to either age or SRQ scores.

\section{Baseline data}

No significant differences were found between the participants in the three treatment groups with respect to demographics, diagnosis or antidepressant treatment (Table 1). However, social functioning as measured by the social functioning scale specifically developed for British Pakistani women ${ }^{21}$ was significantly lower in the antidepressant group than in the other two groups (Table 2).

\section{Treatment adherence}

In the social intervention group, 27 participants (69\%) attended eight or more of the ten sessions, 9 participants attended four to seven sessions, 1 participant attended only once, and 2 participants did not attend any. One of these two women was in
Pakistan and the other did not give a reason. In the combined treatment group, 18 participants (43\%) attended eight or more sessions, 12 attended three to seven sessions, 1 attended twice, 1 attended once, and 10 did not attend any. Of these ten, three refused or were unable to attend, two were in Pakistan, one had moved from the area, and four did not give a reason. The number of sessions attended was significantly higher in the social intervention group (Mann-Whitney $P=0.050$ ).

At 3 months, 21 participants ( $54 \%$ of those with follow-up data) in the antidepressant group and $24(71 \%)$ in the combined treatment group were being prescribed antidepressants compared with $6(18 \%)$ of those receiving social intervention alone $\left(\chi^{2}=19.2\right.$, d.f. $=2, P<0.001)$. At 9 months, antidepressants were being prescribed to ten individuals (26\%) in the antidepressant group, 7 $(21 \%)$ in the combined treatment group, and $6(18 \%)$ in the social intervention group $\left(\chi^{2}=0.6\right.$, d.f. $\left.=2, P<0.73\right)$.

\section{Primary outcomes}

The reduction in the HRSD score was greater in the social intervention and combined treatment groups than the antidepressant group at both 3 and 9 months, but both of these differences fell short of statistical significance (Table 3). The change in HRSD score for the combined treatment group was not significantly different from that in the social intervention group at either 3 or 9 months. Allowance for missing follow-up data did not alter these findings.

\section{Secondary outcomes}

There was a greater increase in social functioning in the social intervention group and the combined treatment group than in the antidepressant group at both 3 and 9 months, but these were significant only at 3 months (Table 3 ). Satisfaction scores were significantly greater in the social intervention and combined treatment group compared with the antidepressant group at both 3 and 9 months. The combined treatment and social intervention groups were not significantly different in change of any of these scores at 3 and 9 months. Allowance for missing follow-up data did not alter these findings.

\section{Remission of depression}

There was no significant difference between the three groups with respect to the number of participants for whom depression had remitted as judged by a non-depression CIS-R diagnosis at 9 months (antidepressants: 8/30 (27\%); social intervention: 11/37 (30\%); combined treatment: $\left.12 / 33(36 \%) ; \chi^{2}=0.7, P=0.69\right)$.

At 3 months, eight participants in total had achieved remission defined by HRSD score $\leqslant 7$ : four in the antidepressant group, none in the social intervention group and four in the combined treatment group. At 6 months, 14 participants had achieved remission: 6 in the antidepressant group, 2 in the social intervention group and 6 in the combined treatment group.

\section{Analysis by patient rather than by practice}

The data (Fig. 2) suggest a more rapid reduction of HRSD score in the social intervention group than in those treated with antidepressants alone. Ignoring the fact that patients were randomised in clusters defined by their GP, this difference is significant $(P=0.018)$ at 3 months, but not 9 months $(P=1.0)$. The combined treatment group also shows a significantly greater improvement than the antidepressant group at 3 months $(P=0.006)$, but the difference between social intervention and combined treatment groups was not significant $(P=1.0)$. At 9 months there was no significant difference between any of the three groups $(P=0.20)$. 


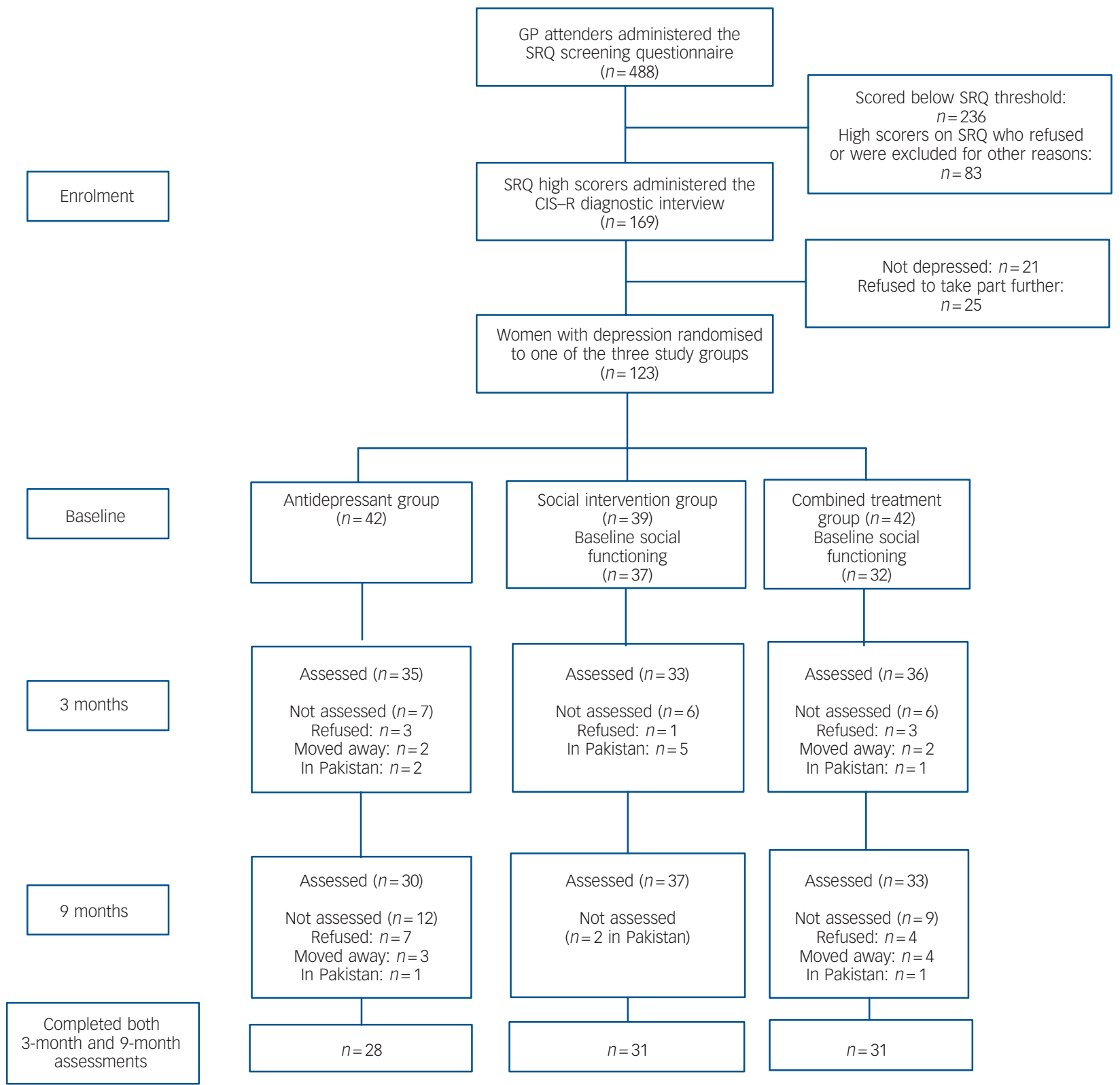

Fig. 1 Study flow chart. Ten patients in total completed assessments at 6 months, but not at 3 months, and 14 completed at 3 months, but not at 6 months.

\section{Qualitative participant feedback}

Barriers and facilitators to participation

A major hindrance to social group participation in the present study was resistance from family members, particularly husbands. The family often did not recognise depression as meriting outside help and lacked faith in the appropriateness of the intervention. Participants described their initial apprehension about the nature of the groups and the degree of disclosure that would be expected.

A key factor encouraging attendance was the taxi service with a female transport facilitator together with facilitators' warmth and empathy. Participants felt obliged to attend as the group facilitators had made elaborate arrangements and the participants felt they should not let them down.

\section{Perceptions about the social intervention}

Participants engaged in the trial because of the culturally appropriate format and content of the sessions. They felt very much at home because the multilingual facilitators were warm and understanding, and written materials were available in their own languages.

Many commented that ten sessions were inadequate for their needs and, in future, for the sessions to be spread over a longer period of time to avoid the abrupt ending of the intervention.

\section{Communication with GP and antidepressant use}

Participants expressed mixed views about their respective GPs. In addition to some positive comments, many found their GPs 'lacking time to explain the expected benefits and side-effects of medication'. The latter was a common reason for discontinuation of medication. Written multilingual information about medication and telephone reminders were mentioned by the participants as ways of encouraging adherence.

\section{Positive perceptions about outcomes and benefits}

Participants described their experience as 'relief from worries', 'feeling fresh' and 'better than their expectations'. They enjoyed meeting people, liked being in the company of others and planned 


\begin{tabular}{|c|c|c|c|}
\hline & $\begin{array}{l}\text { Antidepressant group } \\
\qquad(n=42) N(\%)\end{array}$ & $\begin{array}{l}\text { Social intervention group } \\
\qquad(n=39) N(\%)\end{array}$ & $\begin{array}{l}\text { Combined treatment group } \\
\qquad(n=42) N(\%)\end{array}$ \\
\hline \multicolumn{4}{|l|}{ Preferred language } \\
\hline Urdu & $20(48)$ & $16(41)$ & $13(31)$ \\
\hline English & $7(17)$ & $8(21)$ & $7(17)$ \\
\hline Punjabi & $15(36)$ & $15(38)$ & $22(52)$ \\
\hline First-generation Pakistani & $34(81)$ & $32(82)$ & $37(88)$ \\
\hline \multicolumn{4}{|l|}{ Marital status } \\
\hline Single & $1(2)$ & $1(3)$ & $1(2)$ \\
\hline Married/cohabiting & $27(64)$ & $31(80)$ & $33(79)$ \\
\hline Divorced/separated & $9(21)$ & $4(10)$ & $6(14)$ \\
\hline Widowed & $5(12)$ & $3(8)$ & $2(5)$ \\
\hline Housewife & $34(81)$ & $35(90)$ & $38(90)$ \\
\hline 11 or more years of education & $28(67)$ & $27(69)$ & $26(62)$ \\
\hline Close confidante & $33(79)$ & $27(71)$ & $37(88)$ \\
\hline Previously sought help from GP or psychiatrist & $31(74)$ & $20(51)$ & $29(69)$ \\
\hline Previously received medication for psychological problem & $25(60)$ & $17(44)$ & $23(55)$ \\
\hline \multicolumn{4}{|l|}{ Diagnosis } \\
\hline Mixed anxiety and depression & $21(50)$ & $30(77)$ & $22(52)$ \\
\hline Mild depression & $2(5)$ & $2(5)$ & $4(10)$ \\
\hline Moderate depression & $11(26)$ & $7(18)$ & $11(26)$ \\
\hline Severe depression & $8(19)$ & 0 & $5(12)$ \\
\hline Prescribed antidepressant medication at time of recruitment ${ }^{a}$ & $3(7)$ & $4(10)$ & $8(19)$ \\
\hline
\end{tabular}

\begin{tabular}{|c|c|c|c|}
\hline & $\begin{array}{l}\text { Antidepressant group } \\
\qquad(n=42)\end{array}$ & $\begin{array}{l}\text { Social intervention group } \\
\qquad(n=39)\end{array}$ & $\begin{array}{l}\text { Combined treatment group } \\
\qquad(n=42)\end{array}$ \\
\hline Age, years: mean (s.d.) & $41.3(11.0)$ & $41.3(10.4)$ & $42.6(10.3)$ \\
\hline Self-Reporting Questionnaire, mean (s.d.) & $13.6 \quad(3.3)$ & $11.7(3.2)$ & $12.8(3.5)$ \\
\hline Hamilton Rating Scale for Depression, mean (s.d.) & $17.9 \quad(6.9)$ & $19.3(5.0)$ & $20.2(5.6)$ \\
\hline Social functioning, ${ }^{\mathrm{b}}$ mean (s.d.) & $7.1 \quad(6.7)$ & $13.2(7.2)$ & $13.6(8.5)$ \\
\hline
\end{tabular}

\begin{tabular}{|c|c|c|c|c|c|c|c|c|c|c|c|c|}
\hline & \multicolumn{2}{|c|}{$\begin{array}{l}\text { Antidepressant } \\
\text { group } \\
(n=42)\end{array}$} & \multicolumn{2}{|c|}{$\begin{array}{l}\text { Social inter- } \\
\text { vention group } \\
\quad(n=39)\end{array}$} & \multicolumn{2}{|c|}{$\begin{array}{c}\text { Combined } \\
\text { treatment } \\
\text { group }(n=42)\end{array}$} & \multicolumn{2}{|c|}{$\begin{array}{l}\text { Social } \\
\text { intervention } v \text {. } \\
\text { antidepressant }\end{array}$} & \multicolumn{2}{|c|}{$\begin{array}{l}\text { Combined } \\
\text { treatment } v \text {. } \\
\text { antidepressant }\end{array}$} & \multicolumn{2}{|l|}{$\begin{array}{c}\text { Combined } \\
\text { treatment } v \text {. } \\
\text { social intervention }\end{array}$} \\
\hline & $n$ & Mean (s.d.) & $n$ & Mean (s.d.) & $n$ & Mean (s.d.) & Effect $(95 \% \mathrm{Cl})$ & $P$ & Effect $(95 \% \mathrm{Cl})$ & $P$ & Effect $(95 \% \mathrm{Cl})$ & $P$ \\
\hline \multicolumn{13}{|l|}{ HRSD } \\
\hline 3 months & 35 & $16.9(7.0)$ & 33 & $14.3(4.0)$ & 36 & $14.5(5.4)$ & $-3.3(-8.2$ to 1.5$)$ & 0.14 & -3.6 ( -9.3 to 2.2 ) & 0.17 & $-0.20(-3.73$ to 3.32$)$ & 0.89 \\
\hline 9 months & 30 & $14.8(6.9)$ & 37 & $14.7(4.4)$ & 33 & $13.9(6.1)$ & $-0.9(-4.7$ to 3.0$)$ & 0.59 & $-2.1(-5.3$ to 1.0$)$ & 0.14 & $-1.28(-5.74$ to 3.18$)$ & 0.49 \\
\hline \multicolumn{13}{|l|}{$\begin{array}{l}\text { Social } \\
\text { functioning }\end{array}$} \\
\hline 3 months & 35 & $7.0(7.4)$ & 31 & $14.9(8.8)$ & 29 & $13.6(7.8)$ & (1.4 to 10.8$)$ & 0.020 & 5.9 (1.5 to 10.2$)$ & 0.017 & $-0.25(-3.53$ to 3.03$)$ & 0.85 \\
\hline 9 months & 30 & $9.6(7.8)$ & 35 & $14.2(7.4)$ & 28 & $14.3(8.0)$ & $3.2(-5.0$ to 11.5$)$ & 0.36 & $4.0(-0.1$ to 8.1$)$ & 0.055 & 0.77 (-7.69 to 9.23$)$ & 0.82 \\
\hline \multicolumn{13}{|c|}{ Satisfaction total } \\
\hline 3 months & 35 & $13.1(3.4)$ & 33 & $18.0(2.3)$ & 36 & $17.4(3.3)$ & 4.9 (2.9 to 7.0$)$ & 0.002 & 4.3 (2.6 to 6.0$)$ & 0.001 & $-0.64(-3.24$ to 1.96$)$ & 0.55 \\
\hline 9 months & 30 & $12.9(2.8)$ & 36 & $17.6(2.0)$ & 33 & $16.9(2.7)$ & 4.7 (2.6 to 6.7) & 0.002 & 4.0 (1.8 to 6.2 ) & 0.005 & $-0.64(-1.50$ to 0.21$)$ & 0.11 \\
\hline
\end{tabular}

to further continue these new friendships. They were able to confide in others, something they were struggling to do previously at home. They reported that family members and friends noticed a significant difference (e.g. 'friends and family picked up on the improvement').

\section{Discussion}

This is the first reported randomised controlled trial of a social intervention specifically developed for British Pakistani women with depression. For those in the social intervention group, there 


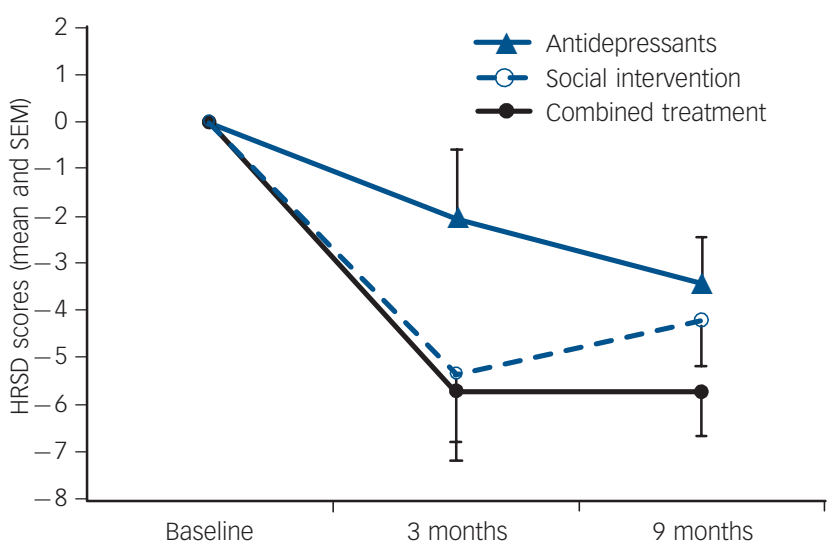

Fig. 2 Adjusted mean Hamilton Rating Scale for Depression (HRSD) scores at baseline, 3 months and 9 months (with robust standard error bars).

were greater improvements in social functioning at 3 months and satisfaction at 3 and 9 months compared with those receiving antidepressants alone. Improvements in depression scores at 3 and 9 months and social functioning at 9 months favoured social intervention over antidepressants alone, but fell short of statistical significance. There were no additional benefits from combining the social intervention with antidepressants over social intervention alone.

Unlike previous trials that have modified existing cognitivebehavioural therapy (CBT) or quality improvement approaches, ${ }^{26,27}$ this new complex social group intervention is guided by our previous research ${ }^{1,2,15}$ highlighting the need for culturally acceptable content and mode of delivery. Individual therapies may be alien to British Pakistani women, whereas the social groups are more akin to their own culture of sharing, doing things together, and mutual social support provided through the extended family and a more communal way of life. Once their initial caution was overcome, attendance at the groups was good, and the women enthusiastically celebrated at the final session. Those in the social intervention group expressed significantly greater satisfaction at 3 months, and continued to feel more satisfied with their care even after the clinical advantages had waned after 9 months.

We successfully developed and delivered the social intervention to this hard-to-reach group of women. The intervention is expensive because of culturally sensitive additions but this is needed to win over participants and families in this population subgroup.

In terms of the comparative efficacy of the social intervention, and in order to inform the design of a future trial, our results suggest some benefit to women with depression at least in the short-term, but there are no clear advantages over antidepressants in the longer term.

\section{Strengths and limitations of the study}

Both in the delivery of the interventions and trial procedures, every effort was made to overcome any barriers. Our social intervention incorporated culturally adapted care and aspects of enhanced interventions similar to those identified by Miranda et $a l^{26}$ as effective components in reducing depression and improving function among ethnic minority women in the USA. The follow-up rates were good: $85 \%$ at 3 months and $81 \%$ at
9 months, and the majority of those lost had actually moved away, often to Pakistan.

Our failure to achieve statistically significant results stems, in part, from our small sample size and the cluster design. The size of this exploratory trial restricted the number of general practices in each study group to two, and for the HRSD there was significant variation between clusters of general practices within study groups compared with the variation within clusters $\left(\mathrm{F}_{(3,117)}=3.9\right.$, $P=0.011)$. Thus cluster randomisation caused considerable loss of power compared with conventional randomisation.

The response to medication may have been limited by adherence. Although we did not directly enquire from the participants about their adherence, at 3 months almost half of the antidepressant group were not being prescribed antidepressants. We encouraged GPs to follow NICE guidelines and participants were instructed to consult their GP for antidepressant prescription. Thus antidepressant prescribing followed usual practice, but other studies have promoted adherence more energetically. Miranda et $a l^{27}$ employed a collaborative care model to support and monitor antidepressant use to achieve greater adherence and better outcomes. It is noteworthy that prescription rates tended to be higher in the combined treatment group, as the psychoeducational session encouraged them to consult their GP. This suggests that a future trial should compare collaborative care alone $v$. antidepressants plus social intervention.

Overall, treatment was not satisfactory as only 14 (15.5\% of those assessed and followed up) reached remission (HRSD $\leqslant 7) .{ }^{28}$ More intensive treatment may be needed such as a longer duration of social intervention and ensuring greater adherence to antidepressants.

\section{Comparison with other studies}

We recruited a cross-section of patients attending general practices, not necessarily patients seeking treatment for depression. However, the mean baseline HRSD of 19.1 falls in the moderately severe range. ${ }^{29}$ This is comparable with cases of depression from inner-city primary care (HRSD 15.2), ${ }^{30}$ and depressed minority women in a US treatment trial (HRSD 16.9). ${ }^{27}$

Mean reductions in HRSD score at 3 months in the current trial for the social intervention and combined treatment groups (5.0 and 5.7 respectively) compares with 3-month HRSD reductions of 4.9 for $\mathrm{CBT}$ and 4.1 for referral to community care reported by Miranda et al. ${ }^{27}$ The reduction in HRSD of 1.3 in our antidepressant group compares unfavourably with the protocolguided antidepressant group in the US study, ${ }^{27}$ in which the HRSD score had fallen by 8.1 at 3 months; this may be explained by the difference in ethnicity and levels of treatment adherence.

\section{Implications}

Participants found the social intervention acceptable with comparative improvement in social functioning and depression. Since depression is very prevalent in this population and treatment is currently very poor, this represents an important development. It is clear that improved antidepressant adherence is needed as this population may be reluctant to use these medications. Participant and facilitator feedback revealed that ten weekly sessions were inadequate and ended abruptly. Outcomes may be improved and more sustained by spreading the groups over a longer period. We also need to ensure initial engagement by explaining the social intervention to the women and their families. 
Richard Gater, MD, FRCPsych, Psychiatry Research Group, University of Manchester, Waquas Waheed, MRCPsych, Lancashire NHS Foundation Trust, Nusrat Husain, $\mathrm{MB}, \mathrm{MPH}, \mathrm{MD}$, Barbara Tomenson, MSC, Saadia Aseem, MPA, Francis Creed, MD, FMedSci, FRCP, FRCPsych, Psychiatry Research Group, University of Manchester, UK

Correspondence: N. Husain, Psychiatry Research Group, Third Floor University Place, Oxford Road, Manchester M13 9PL, UK. Email: nusrat.husain@ manchester.ac.uk

First received 26 Apr 2009, final revision 5 Jan 2010, accepted 28 Jan 2010

\section{Funding}

This study was funded by the Medical Research Council, UK.

\section{References}

1 Gater R, Tomenson B, Percival C, Chaudhry N waheed W, Dunn G, et al. Persistent depressive disorders and social stress in people of Pakistani origin and white Europeans in UK. Soc Psychiatry Psychiatr Epidemiol 2009; 44 198-207.

2 Husain N, Creed F, Tomenson B. Adverse social circumstances and depression in people of Pakistani origin in the UK. Br J Psychiatry 1997; 171: $434-8$.

3 Weich S, Nazroo J, Sproston K, McManus S, Blanchard M, Erens B, et al. Common mental disorders and ethnicity in England: the EMPIRIC study. Psychol Med 2004; 8: 1543-51.

4 Bhui K, Bhugra D, Goldberg D, Dunn G, Desai M. Cultural influences on the prevalence of common mental disorder, general practitioners' assessments and help-seeking among Punjabi and English people visiting their genera practitioner. Psychol Med 2001; 31: 815-25.

5 McKenzie K, Bhui K, Nanchahal K, Blizard B. Suicide rates in people of South Asian origin in England and Wales: 1993-2003. Br J Psychiatry 2008; 193 406-9.

6 Cooper J, Husain N, Webb R, Waheed W, Kapur N, Guthrie E, et al. Self-harm in the UK: differences between South Asians and Whites in rates, characteristics, provision of service and repetition. Soc Psychiatry Psychiatr Epidemiol 2006; 41: 782-8.

7 Khan F, Waheed W. Suicide and self-harm in South Asian immigrants. Psychiatry 2009; 8: 261-4.

8 Commander MJ, Odell SM, Surtees PG, Sashidharan SP. Care pathways for south Asian and white people with depressive and anxiety disorders in the community. Soc Psychiatry Psychiatr Epidemiol 2004; 39: 259-64.

9 Cornwell J, Hull S. Do GPs prescribe antidepressants differently for South Asian patients? Fam Pract 1998; 15: S16-8.

10 Hull SA, Aquino $P$, Cotter S. Explaining variation in antidepressant prescribing rates in east London: a cross sectional study. Fam Pract 2005; 22: 37-42.

11 Bhugra D, Baldwin D, Desai M. A pilot study of the impact of fact sheets and guided discussion on knowledge and attitudes regarding depression in an ethnic minority sample. Prim Care Psychiat 1997; 3: 135-40.

12 Jacob KS, Bhugra D, Mann AH. A randomised controlled trial of an educational intervention for depression among Asian women in primary care in the United Kingdom. Int J Soc Psychiatry 2002; 48: 139-48.
13 Bhugra D, Hicks MH. Effect of an educational pamphlet on help-seeking attitudes for depression among British South Asian women. Psychiatr Serv 2004: 55: 827-9.

14 Campbell $M$, Fitzpatrick $R$, Haines $A$, Kinmonth $A L$, Sandercock $P$, Spiegelhalter $\mathrm{D}$, et al. Framework for design and evaluation of complex interventions to improve health. BMJ 2000; 321: 694-6.

15 Chaudhry N, Waheed W, Husain N, Hijazi S, Creed F. Development and pilot testing of a social intervention for depressed women of Pakistani family origin in the UK. J Ment Health 2009; 18: 504-9.

16 World Health Organisation. A User's Guide to the Self-reporting Questionnaire (SRQ). Division of Mental Health, WHO, 1994.

17 Husain N, Gater R, Tomenson B, Creed F. Comparison of the Personal Health Questionnaire and the Self Reporting Questionnaire in rural Pakistan. J Pak Med Assoc 2006; 56: 366-70.

18 Lewis G, Pelosi AJ, Araya R, Dunn G. Measuring psychiatric disorder in the community: a standardized assessment for use by lay interviewers. Psychol Med 1992; 22: 465-86.

19 National Institute for Health and Clinical Excellence. Depression Management of Depression in Primary and Secondary Care (Clinical Guideline 23). NICE, 2004

20 Hamilton $M$. Development of a rating scale for primary depressive illness. Br J SOC Clin Psychol 1967; 6: 278-96.

21 Bolton $\mathrm{P}$, Tang AM. An alternative approach to cross-cultural function assessment. Soc Psychiatry Psychiatr Epidemiol 2002; 37: 537-43.

22 Ruggeri M, Dall'Agnola R. The development and use of the Verona Expectations for Care Scale (VECS) and the Verona Service Satisfaction Scale (VSSS) for measuring expectations and satisfaction with community-based psychiatric services in patients, relatives and professionals. Psychol Med 1993; 23: 511-23.

23 Rahman A, Iqbal Z, Waheed W, Hussain N. Translation and cultural adaptation of health questionnaires. J Pak Med Assoc 2003; 53: 142-7.

24 Campbell MK, Elbourne DR, Altman DG. CONSORT statement: extension to cluster randomised trials. BMJ 2004; 328: 702-8.

25 Dunn G, Maracy M, Dowrick C, Ayuso-Mateos JL, Dalgard OS, Page H, et al. Estimating psychological treatment effects from a randomised controlled trial with both non-compliance and loss to follow-up. Br J Psychiatry 2003; 183: 323-31

26 Miranda J, Duan N, Sherbourne C, Schoenbaum M, Lagomasino I, JacksonTriche $\mathrm{M}$, et al. Improving care for minorities: can quality improvement interventions improve care and outcomes for depressed minorities? Results of a randomized, controlled trial. Health Serv Res 2003: 38: 613-30.

27 Miranda J, Chung JY, Green BL, Krupnick J, Siddique J, Revicki DA, et al. Treating depression in predominantly low-income young minority women - a randomized controlled trial. JAMA 2003; 290: 57-65.

28 Keller MB. Past, present, and future directions for defining optimal treatment outcome in depression: remission and beyond. JAMA 2003; 289: 3152-60.

29 Yonkers KA, Samson J. Mood disorders measures. In Handbook of Psychiatric Measures (eds AJ Rush, HA Pincus, MB First, D Blacker): 515-48. American Psychiatric Association, 2000

30 Ronalds $\mathrm{C}$, Creed F, Stone $\mathrm{K}$, Webb S, Tomenson B. Outcome of anxiety and depressive disorders in primary care. Br J Psychiatry 1997; 171: 427-33. 